\title{
Cell Surface Area Regulation Under Conditions of Transient OGD in Hippocampal Slice Culture.
}

\author{
Natalya Shulyakova*, Linda. R. Mills* \\ * University Health Network, Toronto Western Hospital, 11-430, 399 Bathurst St., Toronto, ON, \\ Canada, M5T 2S8 and Department of Physiology, University of Toronto, Toronto, ON, Canada.
}

Neurons swell in response to a variety of insults. The capacity to recover i.e., to shrink, after swelling, is clearly critical for neuronal function and survival, but little is known about how this process is regulated. Previous studies in dissociated cultures showed that when neurons shrink they reorganize the plasma membrane making transient vacuole-like dilations (VLDs) to accommodate the decrease in cell surface area [1-3]. This cell surface area regulation (SAR) depends upon the cortical actin cytoskeleton, and is regulated in part by ATP. We hypothesized that neurons in 3D tissues are capable of SAR and that oxygen glucose deprivation (OGD) impairs SAR. To monitor SAR in a 3D tissue we used a Helios gene gun to biolistically transfect hippocampal slice cultures with EGFP targeted to the cytoplasm, or membrane targeted GFP (lckGFP). Transfection rates, 1050 cells/slice, were optimal for imaging individual, non-overlapping neurons (Fig. 1). EGFP transfected neurons were uniformly labeled, while lckGFP transfected cells showed intense labeling in the soma, due to accumulation of membrane bound organelles (Fig. 1B,C). To induce OGD slices were washed $3 \mathrm{X}$ in glucose-free balanced salt solution plus 2mM 2-deoxy-D-glucose and perfused with $5 \% \mathrm{~N}_{2} / 95 \% \mathrm{CO}_{2}$ for 15 or $30 \mathrm{~min}$. Slices were imaged prior, and immediately post OGD, and at 0,7 , and $24 \mathrm{hr}$. post reperfusion.

In cells where the plasma membrane was labeled swelling was apparent immediately post OGD but in cells where the cytoplasm was labeled swelling was significantly delayed and only detected $7 \mathrm{hrs}$. post reperfusion (Fig. 2). This difference suggests that the plasma membrane is a more sensitive indicator of cell swelling than changes in cytoplasmic volume. Both EGFP and lckGFP positive neurons formed VLD-like structures indicating that neurons in slice cultures demonstrate SAR. Neurons swelled and shrank repeatedly in response to cycles (Fig. 3) of osmotic perturbation (normoosmotic-hypoosmotic-normoosmotic) in contrast to previous lower resolution studies on slices which showed neuronal swelling but no recovery (shrinking) post-reperfusion [4]. This difference may be due to our methods which can detect changes in the plasma membrane or cytoplasm in individual cells. OGD did not alter neuronal swelling and shrinking in response to osmotic perturbations (Fig. 4). Our data indicates that neurons in a complex 3D environment demonstrate SAR and that transient OGD does not impair neuronal capacity for SAR. These results suggests SAR is an intrinsic property of neurons in vivo and that neuronal SAR may play an important role in the brain's response to ischemic insults. Supported by NSERC \& CIHR to LRM and MSA to NS.

References:

1. T. L. Herring et al., J.Membr.Biol., 171 (1999) 151.

2. L.R. Mills and C.E. Morris, J.Membr.Biol., 166 (1998) 223.

3. C.E. Morris and U. Homann, J.Membr.Biol., 179 (2001)79.

4. R. Nakajima et al., J. Neurosci. Res., 76 (2004) 723. 

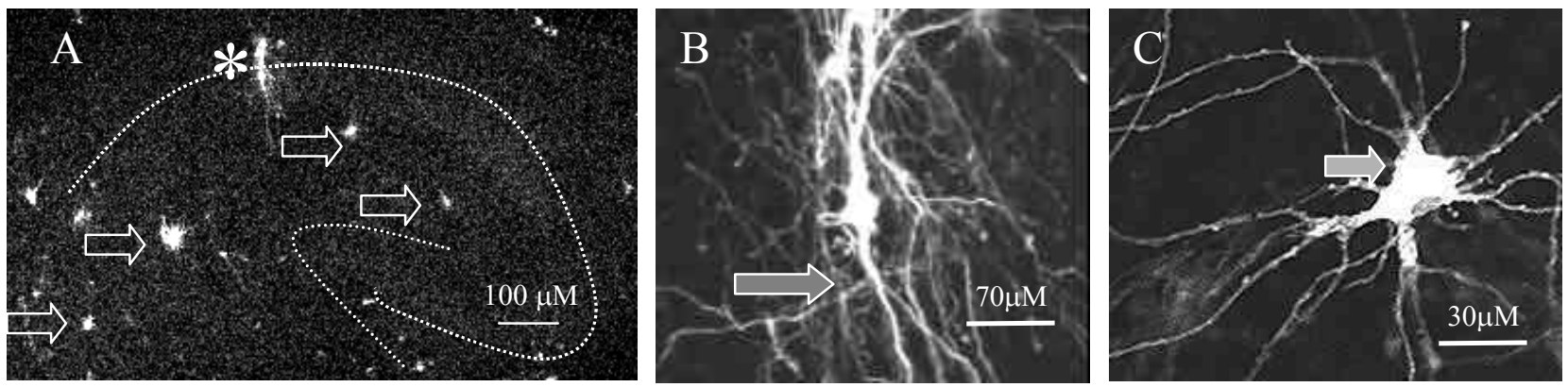

Fig.1: A, EGFP transfection labels the cytoplasm of numerous cells (arrows) in hippocampal slice. * labels a neuron in CA2. B, EGFP labels the cytoplasm so the cell body and neurites (arrow) are readily visible. $\mathrm{C}$, Transfection with lckGFP labels organelle membranes and the plasma membrane; areas where organelles accumulate (arrow at soma) show intense fluorescence. In $\mathrm{B}$, and $\mathrm{C}$ images from 2 different focal planes have been merged to show more details of neuronal processes.
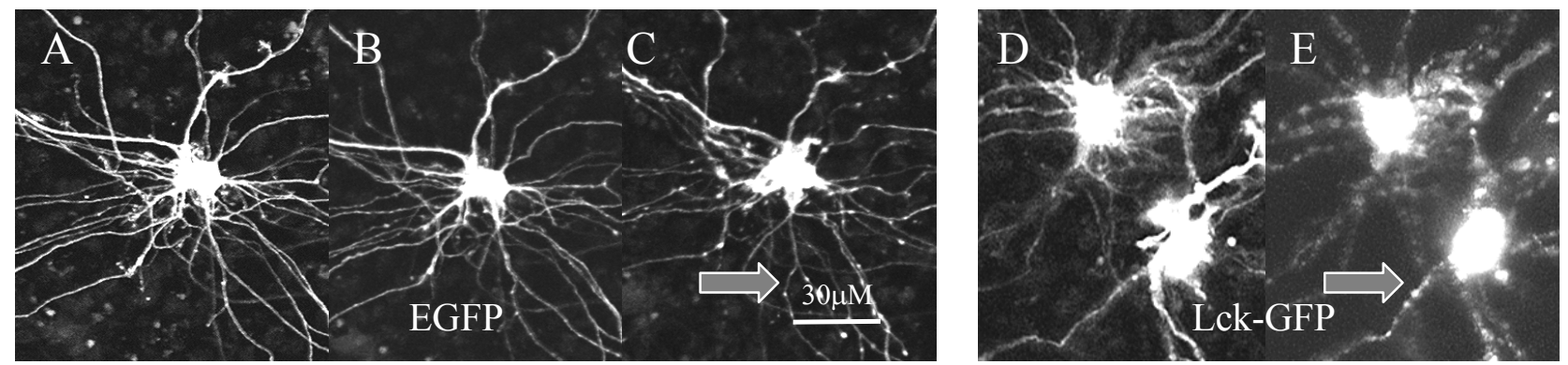

Fig.2: OGD induced changes in hippocampal neurons. A, EGFP+ neuron pre OGD. This cell showed no changes immediately post $15 \mathrm{~min}$. OGD (not shown), or immediately post reperfusion, see B. By C, 7hrs later, the swollen soma and blebbed neurites (arrow) are visible. D, lckGFP+ neuron pre OGD. E, Cell swelling and blebbing are apparent immediately post OGD.

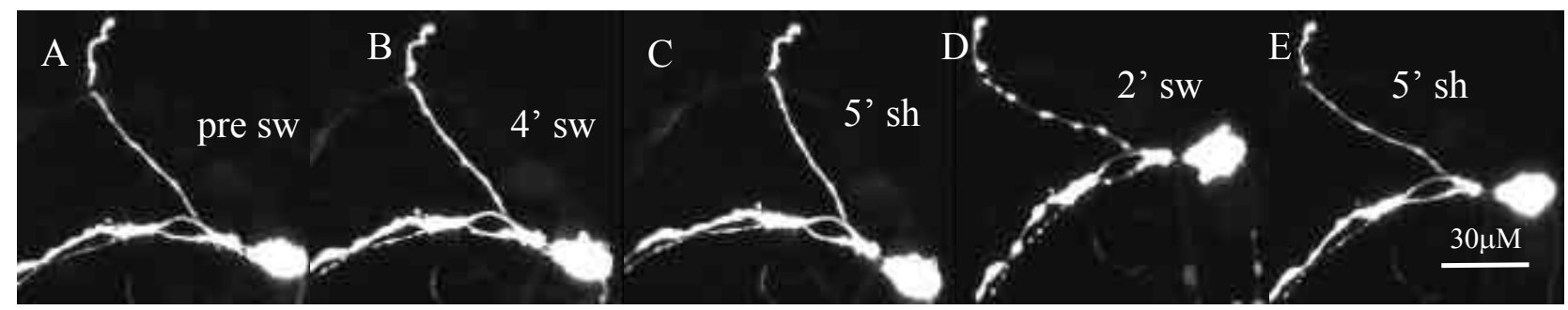

Fig.3: EGFP+ neuron swelling and shrinking in response to 2 cycles of osmotic perturbation. AC, cycle 1. D-E, cycle 2, times shown are minutes in swelling (sw) or shrinking (sh) solutions.

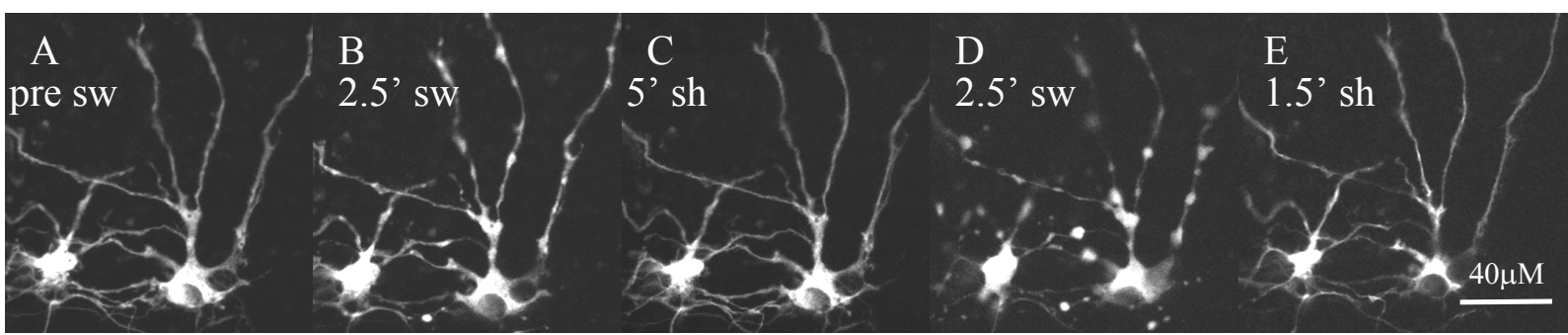

Fig.4: EGFP+ neuron exposed to 2 cycles of swelling and shrinking after a prior 30 min OGD challenge, and subsequent reperfusion. A, 1 min post reperfusion. E, note marked cell shrinking. 